\title{
BRAF V600 mutation profiling in primary skin nodular melanoma in Indonesia: an analysis using high resolution pyrosequencing
}

Hanggoro Tri Rinonce ( $\nabla$ hanggoro_rinonce@ugm.ac.id )

Universitas Gadjah Mada

Rovi Panji Mustiko Aji

Department of Anatomical Pathology, Faculty of Medicine, Public Health, and Nursing, Universitas Gadjah Mada/ Dr. Sardjito Hospital, Sleman, Yogyakarta 55281, Indonesia

Ni'mah Hayati

Department of Anatomical Pathology, Faculty of Medicine, Public Health, and Nursing, Universitas Gadjah Mada/ Dr. Sardjito Hospital, Sleman, Yogyakarta 55281, Indonesia

\section{Maria Fransiska Pudjohartono}

Department of Anatomical Pathology, Faculty of Medicine, Public Health, and Nursing, Universitas Gadjah Mada/ Dr. Sardjito Hospital, Sleman, Yogyakarta 55281, Indonesia

\section{Bidari Kameswari}

Department of Anatomical Pathology, dr. Soeradji Tirtonegoro, Klaten, Central Java 57424, Indonesia

\section{Sumadi Lukman Anwar}

Department of Surgery, Faculty of Medicine, Public Health, and Nursing, Universitas Gadjah Mada/ Dr. Sardjito Hospital, Sleman, Yogyakarta 55281, Indonesia

- Irianiwati

Department of Anatomical Pathology, Faculty of Medicine, Public Health, and Nursing, Universitas Gadjah Mada/ Dr. Sardjito Hospital, Sleman, Yogyakarta 55281, Indonesia

\section{Research note}

Keywords: melanoma, skin cancer, BRAF, pyrosequencing, Indonesia

Posted Date: January 9th, 2020

DOI: https://doi.org/10.21203/rs.2.20532/v1

License: (c) (i) This work is licensed under a Creative Commons Attribution 4.0 International License. Read Full License 
Version of Record: A version of this preprint was published at BMC Research Notes on March 18th, 2020. See the published version at https://doi.org/10.1186/s13104-020-05000-w. 


\section{Abstract}

Objective : We aimed to investigate the prevalence and type of BRAF V600 mutations and the associations with clinicopathological variables in primary skin nodular melanoma cases in Yogyakarta and Central Java, Indonesia using pyrosequencing.

Results : BRAF V600 mutations of the V600E type were found in 21 (53.85\%) specimens. The variant allele frequencies (VAFs) ranged from $5.07 \%$ to $94.70 \%$, averaging $29.05 \%$. However, most cases had low VAFs, with 13 (61.9\%) specimens below $20 \%$ and 4 (19.05\%) below $10 \%$.

\section{Introduction}

Despite its low incidence, melanoma remains the deadliest skin malignancy and the main contributor to deaths from skin malignancies. With a death toll over 50,000 deaths a year, this disease is responsible for over half the mortality from skin cancer (1). The dismal outcomes for melanoma patients call for further research to improve their management, which is currently focused on molecular profiling of melanomas.

The BRAF V600 is the most commonly found somatic mutation in melanomas and thus has become a potential marker and therapeutic target (2). There is a wide range of research on this mutation, from its role in melanoma pathogenesis to the development of specific targeted therapy. Even nowadays, application of the BRAF V600 mutation for diagnostic and prognostic purposes is still under investigation. However, not all countries are equally represented in these bodies of data. For example, there is a paucity of studies from Asian and African countries, in which patterns of clinical behavior and underlying molecular pathogenesis are relatively different from Caucasians.

The under-representation of Asian populations is an important issue to address in melanoma research. Asia accounts for $18.6 \%$ of deaths caused by melanomas, despite only providing $7.6 \%$ of new cases worldwide (3). Asian melanoma cases differ in characteristics from Caucasians, such as predominance of the acral lentiginous subtype and low BRAF mutation prevalence. Variations also exist within certain parts of Asia and even between different ethnicities $(4,5)$.

Melanomas in Indonesia present distinct clinical features, showing different clinical presentations and histopathological subtypes from other Asian populations. As opposed to the acral lentiginous melanoma subtype predominance in Asia, most cases from Indonesia are of the nodular subtype, which is associated with worse prognosis (6). The prevalence of BRAF V600 mutations in melanoma patients from Indonesia has also been shown to be relatively low. In our previous study, we investigated the prevalence of the BRAF V600 mutation among Indonesian melanoma cases using real-time polymerase chain reaction (RT-PCR) as the detection method and obtained a low percentage compared to Asia and other countries (7). To our knowledge, previous researches in Indonesian populations have only used immunohistochemistry or RT-PCR methods in their studies $(7,8)$. With clinical consideration that BRAF inhibitors are indicated for all BRAF V600 mutation-positive tumors, regardless of percentage, the detection of BRAF mutations must be done with more sensitive testing methods. 
Therefore, we aimed to investigate the prevalence and type of BRAF V600 mutations using pyrosequencing in primary skin nodular melanoma in Yogyakarta and Central Java, Indonesia. With the higher sensitivity of this method, more patients with BRAF mutations might benefit from targeted therapy.

\section{Materials And Methods}

This retrospective cross-sectional study was done at the Department of Anatomical Pathology Dr. Sardjito Hospital, Yogyakarta and dr. Soeradji Tirtonegoro Hospital, Central Java, Indonesia. Both hospitals were the main referral hospitals in Yogyakarta Province and Central Java Province, Indonesia. Paraffin-embedded tissue specimens from primary skin nodular melanoma cases in 2011-2018 were used as samples. Thirty-nine specimens from Javanese patients were included in analysis. DNA extraction from formalin-fixed paraffin-embedded (FFPE) primary skin nodular melanoma tissue was performed after selection of tumor-rich slides using the GeneAII® ExgeneTM DNA Extraction Kit (GeneAll Biotechnology, Seoul, Korea) according to the protocol provided by the producer.

We assessed BRAF V600 mutation status using pyrosequencing. Each $25 \mathrm{ng}$ sample of DNA was amplified using 1x PCR buffer (Invitrogen, Frankfurt, Germany), $1.5 \mathrm{mM} \mathrm{MgCl}_{2}, 200 \mu \mathrm{M}$ dNTPs, $0.5 \mathrm{U}$ HotStart Taq-Polymerase (Platinum Taq ${ }^{\text {'m }}$ DNA polymerase, Invitrogen, Frankfurt, Germany), and a 1:9 mixture of forward (5'-CCTAAACTCTTCATAATGCTTGC-3') and reverse (5'-

GGGACACCGCTGATCGTTTAAACTCAG-3') primers with universal biotinylated primers (5'GGGACACCGCTGATCGTTTA-3'). Thermocycling was performed with preheating $95^{\circ} \mathrm{C}$ for 4 minutes and 40 cycles of $95^{\circ} \mathrm{C}$ for 15 seconds, $60^{\circ} \mathrm{C}$ for 30 seconds, and $72^{\circ} \mathrm{C}$ for 20 seconds, followed by $72^{\circ} \mathrm{C}$ for 3 minutes using Biometra PCR Thermocycler (Analytic Jena AG, Jena, Germany). Pyrosequencing was then performed with PyroMark Gold RQ96 Reagent (QIAGEN GmbH, Hilden, Germany), 1x annealing buffer (200 mmol/L Tris acetate, $50 \mathrm{mmol} / \mathrm{L}$ Mg-acetate) after separation of biotinylated sequence using $3 \mu \mathrm{L}$ streptavidin sepharose (GE Healthcare Life Sciences, Amersham, UK) and binding buffer $(10 \mathrm{mmol} / \mathrm{L}$ tris$\mathrm{HCl}, 2 \mathrm{M} \mathrm{NaCl}, 1 \mathrm{mmol} / \mathrm{L}$ EDTA, $1 \mathrm{ml} / \mathrm{L}$ Tween 20) using PyroMark Q96 Vacuum workstation (QIAGEN $\mathrm{GmbH}$, Hilden, Germany). The pyrosequencing primer was 5'-TGATTTTGGTCTAGCTACAG-3'. Pyrograms and quality control of the samples were analysed using PyroMark Q96 Software ® (QIAGEN GmbH, Hilden, Germany).

Clinicopathologic data were obtained from registry records at the Department of Anatomical Pathology Dr. Sardjito and dr. Soeradji Tirtonegoro Hospitals. The data collected included age, sex, anatomic location, lymph node metastasis, tumor thickness, ulceration, mitotic index, necrosis, lymphovascular invasion, and tumor-infiltrating lymphocytes (TILs). The lymph node metastasis, tumor thickness, ulceration, necrosis, lymphovascular invasion, and TILs were observed from hematoxylin-eosin stained slides, while the mitotic index was calculated from Ki-67 immunohistochemistry-stained slides as previously described in our study (7). We used Ventana Ultra Cell Conditioner 1 solution (Ventana Medical Systems, Tucson, AZ, USA) under $\mathrm{pH}$ 8-9 in 64 minutes on $95^{\circ} \mathrm{C}$ for antigen retrieval and the monoclonal Ki67 antibody (Abcam, Cambridge, MA, USA) for the immunostaining according to the protocol previously 
described in our study (7). The associations between BRAF mutation status and clinicopathologic parameters were analyzed by the chi-square test or Fisher's exact test for categorical variables, and the independent t-tests or Mann-Whitney test for continuous variables.

\section{Results}

The patients' age ranged from 21 to 80 years, with an average of 60.67 years of age. Seventeen (43.59\%) patients were male and twenty-two $(56.41 \%)$ patients were female. Twenty-nine patients $(74.36 \%)$ had tumors on extremities, while $10(24.64 \%)$ had tumors on the trunk or head and neck (centrally located).

Out of the thirty-nine samples, BRAF V600 mutations were found in twenty-one (53.85\%) samples. Variant allele frequencies (VAFs) ranged from $5.07-94.70 \%$, with an average of $29.05 \%$ and standard deviation of $26.77 \%$. Fifteen $(71.4 \%)$ samples had VAFs below $30 \%$, while $13(61.9 \%)$ samples had VAFs below $20 \%$. All mutations were of the V600E subtype. The complete distribution of VAFs is shown in Fig. 1.

The associations between mutation status and the clinicopathologic characteristics are shown in Table 1. No statistically significant associations were found between the BRAF V600 mutation status and the clinicopathological characteristics analyzed. 
Table 1

The association between BRAF V600 mutation status and clinicopathologic characteristics

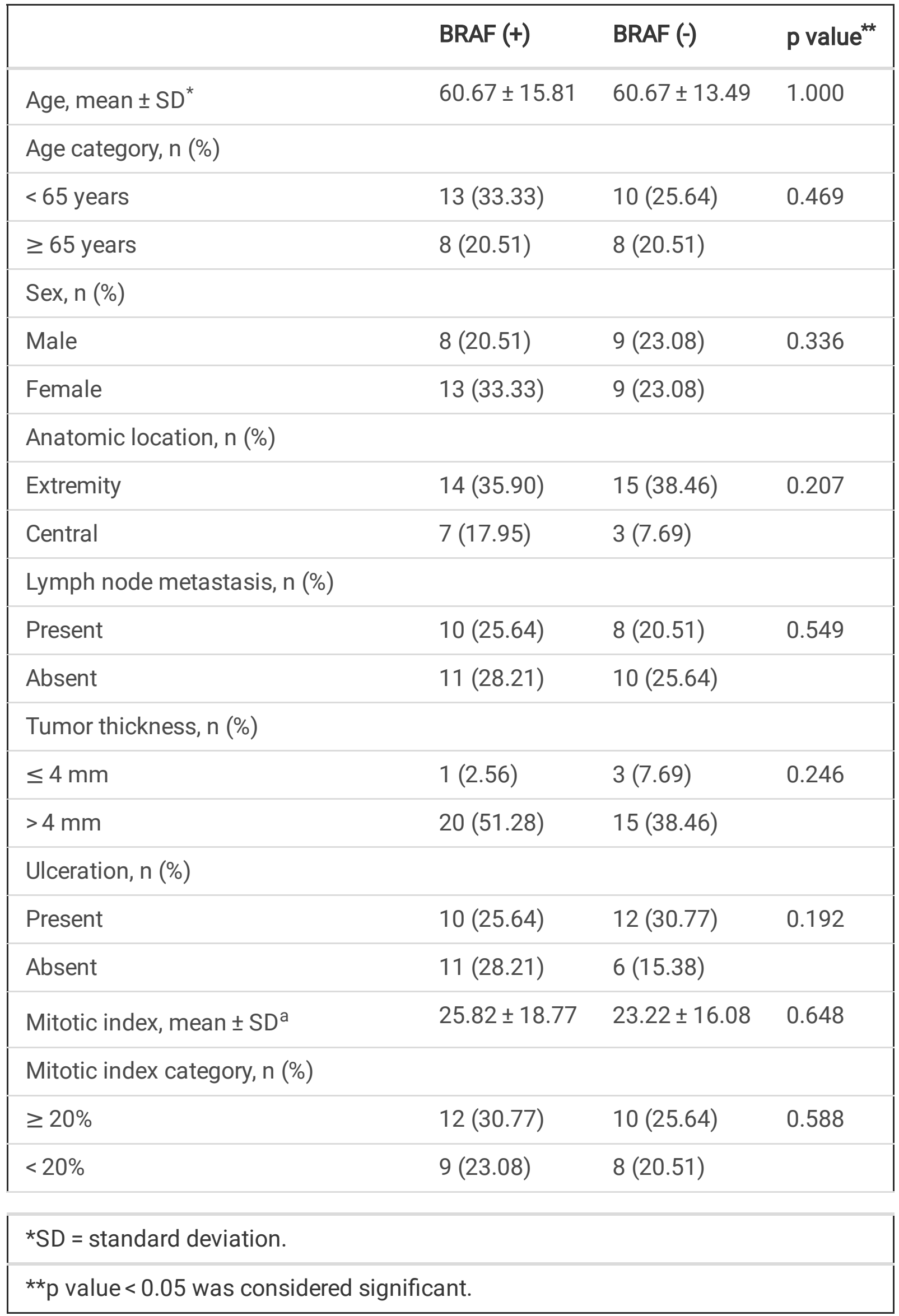




\begin{tabular}{|llll|}
\hline & BRAF (+) & BRAF $(-)$ & p value $^{* *}$ \\
\hline Necrosis, $\mathrm{n}(\%)$ & & & \\
\hline Present & $17(43.59)$ & $11(28.21)$ & 0.570 \\
\hline Absent & $4(10.26)$ & $7(17.95)$ & \\
\hline Lymphovascular invasion, $\mathrm{n}(\%)$ & & & \\
\hline Present & $9(23.08)$ & $4(10.26)$ & 0.153 \\
\hline Absent & $12(30.77)$ & $14(35.90)$ & \\
\hline Tumor-infiltrating lymphocytes, $\mathrm{n}(\%)$ & & & 0.368 \\
\hline Present & $13(33.33)$ & $13(33.33)$ & \\
\hline Absent & $8(20.51)$ & $5(12.82)$ & \\
\hline *SD $=$ standard deviation. & & & \\
\hline ** value<0.05 was considered significant. & & \\
\hline
\end{tabular}

\section{Discussion}

In this study, the BRAF V600 mutation was found in $53.85 \%$ of the nodular melanoma cases. This result resembles the mutation prevalence found in Caucasian populations, which range around $40-60 \%$ (9). Data on the nodular subtype from Asia is scarce with two studies reporting rates of $50 \%$ and $29.4 \%$ from Japan and Turkey, respectively $(10,11)$. The outcomes of our research are especially at odds with the result of our previous study, which report a 10\% BRAF V600 prevalence among Indonesian nodular melanomas.

The higher results found in this study compared to our previous study may arise from the difference in methods. In terms of sensitivity, pyrosequencing is superior compared to high-resolution melt PCR studies (12). In a comparation of methods, RT-PCR detected $98 \%$ of cases while pyrosequencing had $100 \%$ sensitivity (13). This would account for the lower mutation prevalence detected in our previous study with RT-PCR.

Analysis of the VAF in our study shows a wide variation of $5.07-94.70 \%$, with an average of $29.05 \%$. However, most of the cases had low VAFs, with 13 (61.9\%) specimens below $20 \%$ and 4 (19.05\%) below $10 \%$. For comparison, a previous study on BRAF mutation profiling in melanomas report a VAF below $20 \%$ only in $28 \%$ of samples and below $10 \%$ in $13 \%$ (14). Given the dominance of low VAF percentages, many mutation-positive tumors which would have been detectable by pyrosequencing would likely go undetected in RT-PCR studies. 
When compared to other Asian countries, the BRAF V600 prevalence in Indonesia is relatively high. This mutation is found in $11.9-41.8 \%$ of Asian melanoma cases in general $(5,11,15,16)$. The only two studies on Asian nodular melanoma cases reported the mutation prevalence at $29.4 \%$ and $50 \%(10,11)$. This suggests that Indonesian melanoma cases have a high BRAF V600 mutation prevalence, but at low intratumoral mutation rates. Variations in melanoma subtype, ethnicity, and different mutation detection methods may partially be responsible for the differences found.

No significant associations were found with clinicopathologic characteristics. The general trends, such as younger age, central location, absent ulceration, and presence of lymphovascular invasion, mirror the findings of previous Asian studies. In our previous study done with RT-PCR in nodular melanoma patients in Indonesia, central location and lymphovascular invasion were correlated with BRAF V600 mutation. The differences could have been caused by the low VAFs found in this study, which may cause mutationpositive tumor to behave like mutation-negative tumors instead. Another factor that possibly dissipated any correlations would be the unbalanced proportions of patient characteristics, as the subjects in this study were dominated by advanced extremity-located melanomas.

The presence of TILs is one of the indicators of the immune response to melanomas and is associated with better prognosis. Oncogenic BRAF mutations are known to contribute to immune escape of the tumor cells, which may explain the lower presence of TILs in tumors with BRAF mutations (17). Treatment with BRAF inhibitors are known to increase the number of TILs, further reaffirming the connection between BRAF and the immune response to melanoma cells. Along with the other associations, our results suggest that Indonesian nodular melanoma cases with the BRAF mutation tend to have worse prognoses.

BRAF mutation testing and targeted therapy has yet to be used in melanoma case management in Indonesia. These are due to limitations in both availability and costs. Currently, BRAF mutation testing and BRAF inhibitors are not included in the Indonesian national health insurance program. Healthcare centers that provide molecular mutation testing are also still scarce. Most melanoma patients in Indonesia cannot afford the price of completing a whole cycle of BRAF inhibitor therapy. As a result, BRAF V600 mutation testing is only done in research settings and treatment for melanomas is commonly limited to surgery and cytotoxic chemotherapy (dacarbazine).

These difficulties suggest that currently it is only possible to provide molecular testing and treatment to a select portion of patients who would most likely benefit from it. The mutation rate could be a potential marker to predict response to targeted therapy. A study shows that higher BRAF V600 mutation VAFs are associated with better response rates to vemurafenib (18). Quantification of BRAF mutation levels may help select patients with the best potential for molecular treatment.

Another option would be to use a less sensitive method for clinical usage in Indonesia, such as RT-PCR. The facilities for this method are more widely available, easier, and cheaper in Indonesia than pyrosequencing. Although only tumors with higher VAFs would be detected by PCR studies, this would mean that the identified tumors will have better treatment response. Thus, using PCR methods for 
mutation detection may be more feasible and cost-effective for prognostic and therapy decision-making in Indonesia.

Our previous research provided the BRAF V600 mutation prevalence in Indonesian skin nodular melanoma cases. This research improved it using a more sensitive method and specified the mutation subtype. Through these studies, we have advanced knowledge on BRAF V600 mutation profiles in primary skin nodular melanoma cases in Indonesia. This information will help develop prognostication and treatment decision-making for Javanese patients. Further studies are needed regarding the role of BRAF V600 mutation on the prognosis of Indonesian patients.

\section{Conclusion}

The BRAF V600E mutation was found in $53.85 \%$ of primary skin nodular melanomas, albeit with low mutation rates. The high prevalence of mutations suggest that many Indonesian melanoma cases may benefit from molecular testing and targeted therapy, but more sensitive detection methods are needed for BRAF V600 mutation status testing. However, availability and feasibility may need to be considered for usage in clinical practice. Further research is needed on the prognostic value of this mutation in Indonesian populations.

\section{Limitations}

The small sample size was the main limitations in this study. Besides that, all the patients were Javanese people, the largest ethnic group in Indonesia. Data in this study might not be able to represent Indonesian population which consists of more than 600 ethnic groups. Future studies with larger sample sizes from various ethnicities are needed to further improve knowledge on the BRAF V600 mutation profile and associations with clinicopathologic characteristics in Indonesian primary skin melanomas.

\section{Abbreviations}

DNA

Deoxyribonucleic acid

FFPE

Formalin-fixed paraffin-embedded

HRP

Horseradish peroxidase

MAPK

Mitogen-activated protein kinase

RT-PCR

Real-time polymerase chain reaction

TILS

Tumor-infiltrating lymphocytes 
VAFs

Variant allele frequencies

\section{Declarations}

\section{Acknowledgements}

The authors want to thank to Nur Eka Wiraditya (Department of Anatomical Pathology, Faculty of Medicine, Public Health, and Nursing, Universitas Gadjah Mada, Yogyakarta, Indonesia) for his assistance in laboratory works. The pyrosequencing was performed at the Institute of Pathology, Mediziniche Hochschule Hannover, 30625 Hannover, Germany (AG Prof. Ulrich Lehmann).

\section{Funding}

This work was funded by Faculty of Medicine, Public Health, and Nursing, Universitas Gadjah Mada by the grant of Dana Masyarakat. The publication costs are funded by Faculty of Medicine, Public Health and Nursing, Universitas Gadjah Mada.

\section{Availability of data and materials}

All data generated or analyzed during this study are included in the submission. The raw data are available from the corresponding author on reasonable request.

\section{Authors' contributions}

HTR designed the study and performed the analysis. HTR, MFP, SLA, and I wrote the manuscript. RPMA, $\mathrm{NH}$, and BK contributed to the data collecting and analysis. All authors read and approved the final manuscript.

\section{Ethics approval and consent to participate}

This study protocol was reviewed and approved by the Medical and Health Research Ethics Committee of Faculty of Medicine, Public Health, and Nursing, Universitas Gadjah Mada (KE/FK/0759/EC/2019).

\section{Consent for publication}

Not applicable.

\section{Competing interests}

The authors declare that they have no competing interests.

\section{References}


1. Karimkhani C, Green AC, Nijsten T, Weinstock MA, Dellavalle RP, Naghavi M, et al. The global burden of melanoma: results from the Global Burden of Disease Study 2015. Br J Dermatol. 2017;177:13440.

2. Zaman A, Wu W, Bivona TG. Targeting Oncogenic BRAF: Past, Present, and Future. Cancers (Basel). 2019;11(1197):1-19.

3. The Global Cancer Observatory. GLOBOCAN Fact Sheets: Melanoma of skin. 2019.

4. Morita SY, Markovic SN. Molecular targets in melanoma: time for "ethnic personalization." Expert Rev Anticancer Ther. 2012;12(5):601-8.

5. Kang X, Zeng Y, Liang J, Li J, Ren D, Chai L, et al. Aberrations and clinical significance of BRAF in malignant melanoma: A series of 60 cases in Chinese Uyghur. Medicine (Baltimore). 2018;97(1):1-6.

6. Pasaribu ET, Setiaji K, Rinonce HT, Azhar RY, Wahyono A, Pasaribu ET. Clinical and pathological profile of skin melanoma in Dr. Sardjito Hospital, Yogyakarta, Indonesia. Eur J Surg Oncol. 2019:e137. Available from https://doi.org/10.1016/j.ejso.2018.10.466.

7. Rinonce HT, Aji RPM, Hayati N, Pudjohartono MF, Kameswari B, Irianiwati. Low BRAF V600 mutation prevalence in primary skin nodular melanoma in Indonesia: a real-time PCR detection among Javanese patients. BMC Proceedings; 2019;13(15):1-6.

8. 8. Usman HA, Hernowo BSH, Tobing MDL, Hindritiani R. Result analysis of BRAF V600E gene mutation using molecular and immunohistochemistry detection in acral malignant melanoma. Asia Pacific J Cancer Care. 2018;3(3):43-5.

9. 9. Liu Y, Sheikh MS. Melanoma: molecular pathogenesis and theraupetic management. Mol Cell Pharmacol. 2015;6(3):228.

10. 10. Can N, Taştekin E, Yalta TD, Süt N, Korkmaz S, Usta U, et al. BRAF V600 mutation profile of metastatic melanoma in the Thrace Region of Turkey. Turkish J Pathol. 2018;34(2):134-42.

11. 11. Yamazaki N, Tanaka R, Tsutsumida A, Namikawa K, Eguchi H, Omata W, et al. BRAF V600 mutations and pathological features in Japanese melanoma patients. Melanoma Res. 2015;25(1):9-14.

12. 12. Cheng L, Lopez-beltran A, Massari F, Maclennan GT, Montironi R. Molecular testing for BRAF mutations to inform melanoma treatment decisions: a move toward precision medicine. Mod Pathol [Internet]. 2018;31(1):24-38. Available from: http://dx.doi.org/10.1038/modpathol.2017.104

13. 13. Ihle MA, Fassunke J, König K, Grünewald I, Schlaak M, Kreuzberg N, et al. Comparison of high resolution melting analysis, pyrosequencing, next generation sequencing and immunohistochemistry to conventional Sanger sequencing for the detection of p. V600E and non-p. V600E BRAF mutations. BMC Cancer. 2014;14(13):1-13.

14. 14. Lokhandwala PM, Tseng $L$, Rodriguez E, Zheng G, Pallavajjalla A. Clinical mutational profiling and categorization of BRAF mutations in melanomas using next generation sequencing. BMC Cancer. 2019;19(665):1-10.

15. 15. Sheen Y, Liao Y, Liau J, Chu C. Prevalence of BRAF and NRAS mutations in cutaneous melanoma patients in Taiwan. J Formos Med Assoc [Internet]. 2019;115(2):121-7. Available from: 
http://dx.doi.org/10.1016/j.jfma.2015.02.001

16. 16. Jin SA, Chun SM, Choi YD, Kweon S-S, Jung ST, Shim HJ, et al. BRAF mutations and KIT aberrations and their clinicopathological correlation in 202 Korean melanomas. J Invest Dermatol. 2013;133(2):579-82.

17. Fu Q, Chen N, Ge C, Li R, Li Z, Zeng B, et al. Prognostic value of tumor-infiltrating lymphocytes in melanoma: a systematic review and meta-analysis. Oncoimmunology [Internet]. 2019;8(7):1-14. Available from: https://doi.org/10.1080/2162402X.2019.1593806

18. Lebbé C, How-Kit A, Battistella M, Sadoux A, Podgorniak M, Sidina I. BRAF (V600) mutation levels predict response to vemurafenib in metastatic melanoma. Melanoma Res. 2014;24:415-8.

\section{Figures}

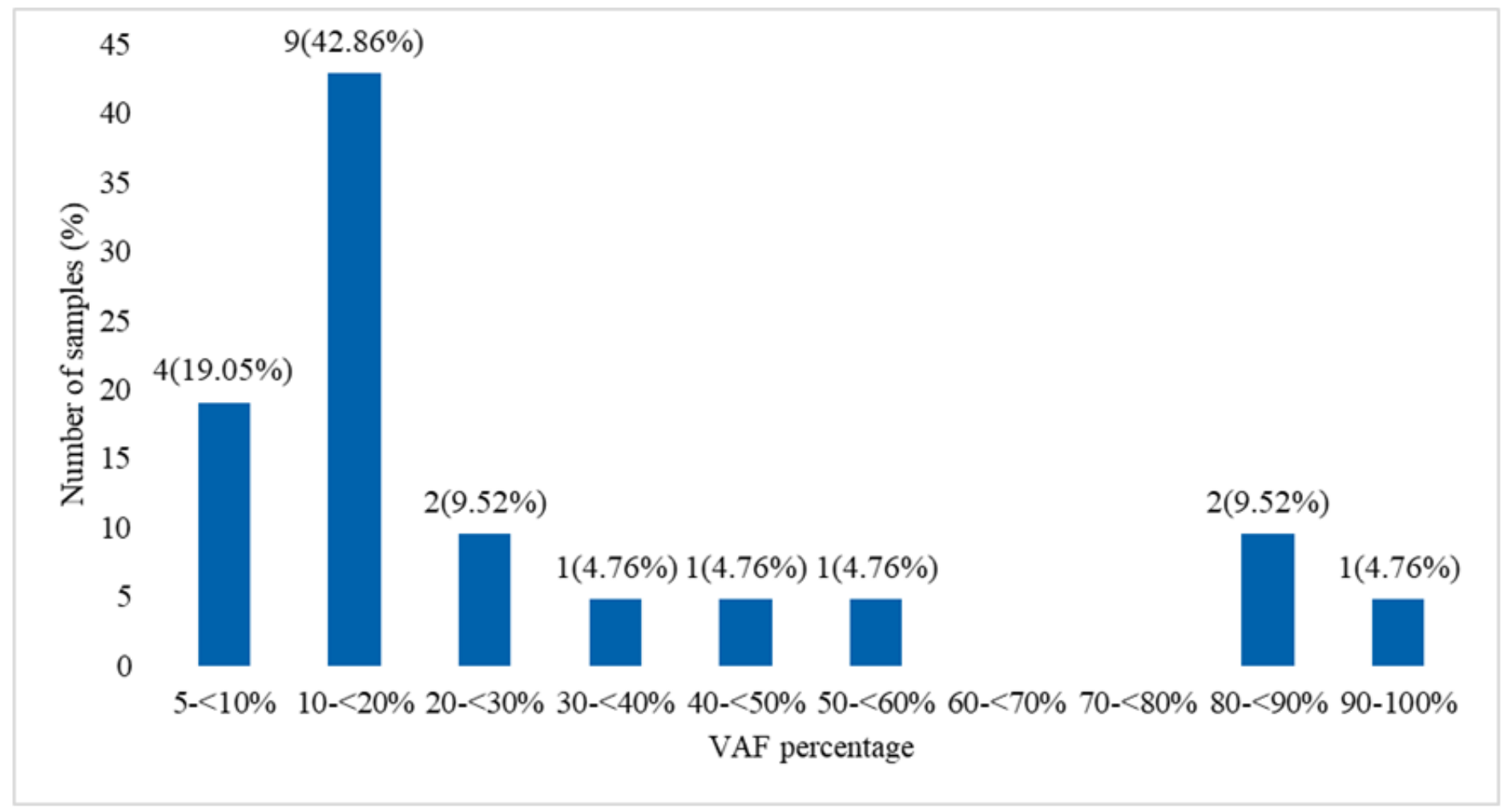

\section{Figure 1}

The distribution of BRAF V600 mutation variant allele frequencies 